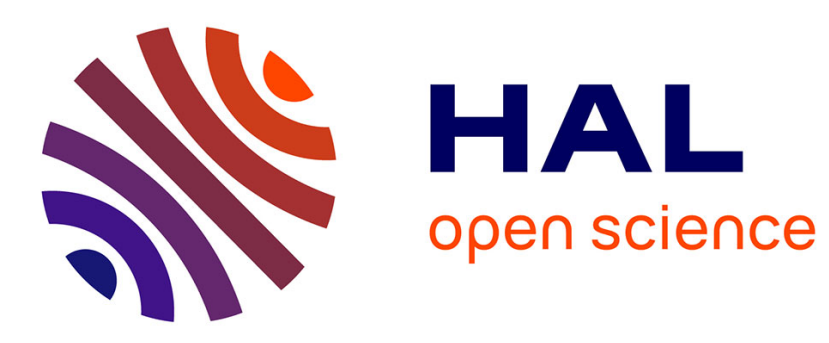

\title{
Carbon monoxide radiation in an equilibrium plasma torch facility
}

Sean Mcguire, Augustin Tibère-Inglesse, Christophe O Laux, Brett A Cruden

\section{To cite this version:}

Sean Mcguire, Augustin Tibère-Inglesse, Christophe O Laux, Brett A Cruden. Carbon monoxide radiation in an equilibrium plasma torch facility. AIAA Scitech 2019 Forum, 2019, San Diego, France. 10.2514/6.2019-1775 . hal-02494095

\section{HAL Id: hal-02494095 https://hal.science/hal-02494095}

Submitted on 28 Feb 2020

HAL is a multi-disciplinary open access archive for the deposit and dissemination of scientific research documents, whether they are published or not. The documents may come from teaching and research institutions in France or abroad, or from public or private research centers.
L'archive ouverte pluridisciplinaire HAL, est destinée au dépôt et à la diffusion de documents scientifiques de niveau recherche, publiés ou non, émanant des établissements d'enseignement et de recherche français ou étrangers, des laboratoires publics ou privés. 


\title{
Carbon monoxide radiation in an equilibrium plasma torch facility
}

\author{
Sean D. McGuire*, Augustin Tibère-Inglesse ${ }^{\dagger}$ and Christophe O. Laux ${ }^{\ddagger}$ \\ Laboratoire EM2C, CNRS UPR288, CentraleSupélec, Université Paris-Saclay \\ 10 rue Joliot-Curie, F-91190, Gif-sur-Yvette, France \\ Brett A. Cruden ${ }^{\S}$ \\ AMA Inc. at NASA Ames Research Center \\ Moffett Field, CA 94035
}

\begin{abstract}
We present spectrally resolved measurements of CO 4th positive radiation down to $170 \mathrm{~nm}$ under equilibrium conditions. The emission is calibrated in absolute intensity by using an argon discharge calibrated to NIST standards. The measurements were undertaken to validate radiation models available in the literature. NASA EAST measurements had noted a discrepancy between radiation model predictions and their shock tube measurements. Our goal was to perform these measurements under equilibrium conditions and compare against model predictions. To that end, we selected several datasets for the electronic transition moment available in the literature. These were used to calculate state-specific Einstein coefficients for use with the radiation code SPECAIR. Three datasets were tested and two were found to give good agreement with the experimental results.
\end{abstract}

\section{Introduction}

In 2013, researchers at NASA Ames performed an experimental study of $\mathrm{CO} 4$ th positive $\left(\mathrm{A}^{1} \Pi \rightarrow \mathrm{X}^{1} \Sigma^{+}, 4+\right)$ in the EAST shock tube facility. ${ }^{1,2}$ Their work was part of an effort to characterize the radiative heat flux under Venus and Mars entry conditions, and to reduce the uncertainty associated with this radiative heat flux. The radiative heat flux is expected to be an important contributor for manned missions beyond Earth orbit, due to the large capsule size and higher entry velocity associated with such missions. ${ }^{2-5}$ In particular, the radiative heat flux is expected to become significant for Mars and Venus entries at speeds above $7.5 \mathrm{~km} / \mathrm{s}$. The Pioneer Venus missions entered at velocities in excess of $11 \mathrm{~km} / \mathrm{s}$ where the radiative heat flux was expected to be important. This will become even more important for the larger capsule sizes used as a part of manned missions. Beyond direct entry scenarios, there is significant interest in using aerocapture maneuvers for missions to Mars and Venus in order to increase the percentage of useful payload. ${ }^{4,6-8}$ Depending upon the specific parameters of this maneuver, the radiative heat flux can represent an important portion of the total heat flux. For Venus and Mars atmospheres, composed primarily of $\mathrm{CO}_{2}$, the $\mathrm{CO}(4+)$ band in the VUV represents either a significant or dominant portion of the radiative heat flux. ${ }^{1,2}$ It is therefore important to validate the radiation models used for this system and to reduce the corresponding uncertainty as much as possible.

The work of Brandis et al. ${ }^{1}$ and Cruden et al. ${ }^{2}$ compared experimental measurements of $\mathrm{CO}(4+)$ radiation with theoretical predictions made using the HARA, ${ }^{9,10}$ HyperRad and NEQAIR ${ }^{11}$ radiation codes. Their goal was to validate the radiation codes used to compute the $\mathrm{CO}(4+)$ emission in the VUV. When the equilibrium post-shock temperature was used, the models underestimated the measured radiation by a factor of 2 . They noted that a large portion of the $\mathrm{CO}(4+)$ spectrum was blackbody limited. Fitting a blackbody curve to this portion of the spectrum yielded a second estimate of temperature, found to be between $2-9 \%$ higher than the equilibrium shock calculation. By using this second temperature, they achieved generally good agreement between the radiation models and the

*Assistant Professor, Laboratoire EM2C, CNRS UPR288, CentraleSupélec, Université Paris Saclay, AIAA Member

† Graduate Student, CentraleSupélec

†Professor, Laboratoire EM2C, CNRS UPR288, CentraleSupélec, Université Paris Saclay, AIAA Associate Fellow

${ }^{\S}$ Sr. Research Scientist, Aerothermodynamics Branch, NASA Ames Research Center, MS 230-2, Moffett Field, CA, AIAA Associate Fellow 
measurements. However, discrepancies remained and there continued to be the uncertainty as to whether or not the flow was in equilibrium, or had at least reached a steady-state temperature. This prevented them from concluding on the validity of the radiation models. Our goal is to address this issue. The plasma torch facility at laboratoire EM2C operates at atmospheric pressure and provides a plasma in chemical and thermodynamic equilibrium at the nozzle exit. We therefore have the capability to reduce the specific uncertainty encountered by Brandis et al and to make measurements of $\mathrm{CO}(4+)$ emission at a known temperature. A specific objective of this paper is to compare the performance of three $\mathrm{CO}$ radiation models via direct comparison with the experimental data obtained in the VUV. The three models studies use transition probabilities based on the electronic transition moment functions of DeLeon, ${ }^{12,13}$ Kirby and Cooper ${ }^{14}$ and Spielfiedel et al. ${ }^{15}$

\section{Theory}

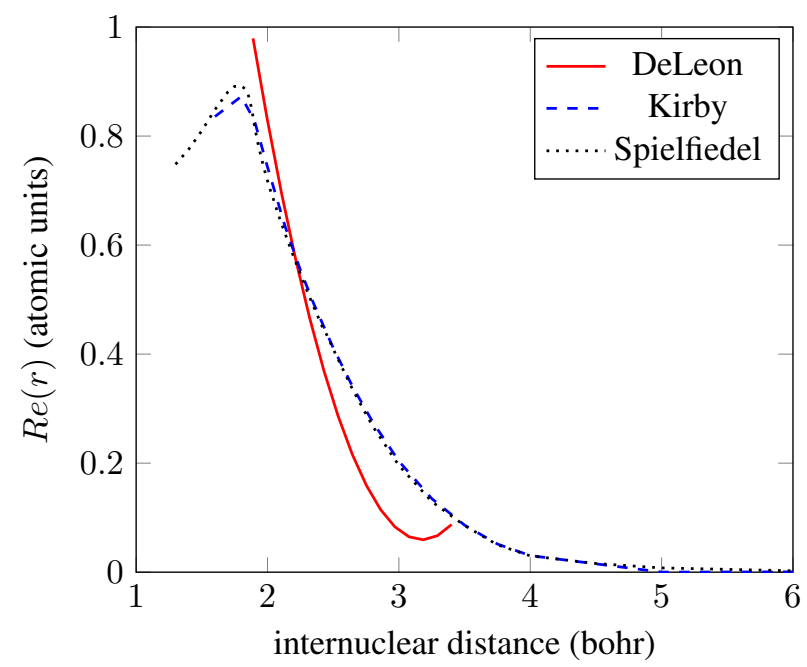

Figure 1: $R e(r)$ values taken from DeLeon, ${ }^{12,13}$ Kirby and Cooper ${ }^{14}$ and Spielfiedel et al. ${ }^{15}$

For the analysis presented here, all experimental results are compared with the SPECAIR radiation model. SPECAIR was originally based upon the NEQAIR radiation code. Details of SPECAIR are provided in the literature. ${ }^{16,17}$ The treatment used here is detailed in Refs. $[16,18]$. The spectral location of each feature is calculated from the Dunham expansion coefficients taken from Farrenq et al ${ }^{19}$ and Simmons et al. ${ }^{20}$ The Einstein coefficients for each rovibrational transition are calculated based upon the following expression:

$$
A_{\nu^{\prime}, J^{\prime} \rightarrow \nu^{\prime \prime}, J^{\prime \prime}}=\frac{64 \pi^{4} \nu^{3}}{3 h c^{3}} R e_{\nu^{\prime}, \nu^{\prime \prime}}^{2} \frac{S_{J^{\prime \prime} \Lambda^{\prime \prime}}^{J^{\prime} \Lambda^{\prime}}}{2 J^{\prime}+1}
$$

where:

$$
R e_{\nu^{\prime}, \nu^{\prime \prime}}^{2}=\left[\int \psi_{\nu^{\prime}} \operatorname{Re} \psi_{\nu^{\prime \prime}}\right]^{2}
$$

$R e_{\nu^{\prime}, \nu^{\prime \prime}}$ is the electronic-vibrational transition moment. $\psi_{\nu^{\prime}}$ and $\psi_{\nu^{\prime \prime}}$ represent the vibrational wavefunctions of the upper and lower states respectively. $S_{J^{\prime \prime} \Lambda^{\prime \prime}}^{J^{\prime} \Lambda^{\prime}}$ is the Hönl-London factor. $\nu$ and $J$ represent the vibrational and rotational quantum numbers with ' and " representing the upper and lower states, respectively. For these calculations, the Rydberg-Klein-Rees (RKR) approximation was first used to determine the potential energy curves for both the X and A states of CO. The corresponding vibrational wavefunctions were then calculated. Finally, the electronic transition moment $R e(r)$ was taken from one of three datasets reported in the literature: DeLeon, ${ }^{12,13}$ Kirby and Cooper ${ }^{14}$ and Spielfiedel et al. ${ }^{15}$ DeLeon supplies an interpolation based on his experimental data, and those previously reported by Field et al. ${ }^{21}$ The models of Kirby and Spielfiedel rely on ab initio calculations. All three datasets will be used to compute emission spectra for comparison with our measured spectra. Figure 1 shows a comparison of the three electronic transition moments. DeLeon supplies experimental measurements of $\operatorname{Re}(r)$, based upon a compilation of their LIF measurements and previous values reported by Field et al. ${ }^{21}$ They supply an experimental fit over the limited 
range of internuclear distances reproduced in the figure. Also, the data of Kirby and Cooper have been divided by a factor of $\sqrt{2}$ - this is required due to the conventions and definitions used in their paper. All data have all been converted to atomic units. The ab initio calculations of Kirby and Spielfiedel are quite close - to within 5\%.

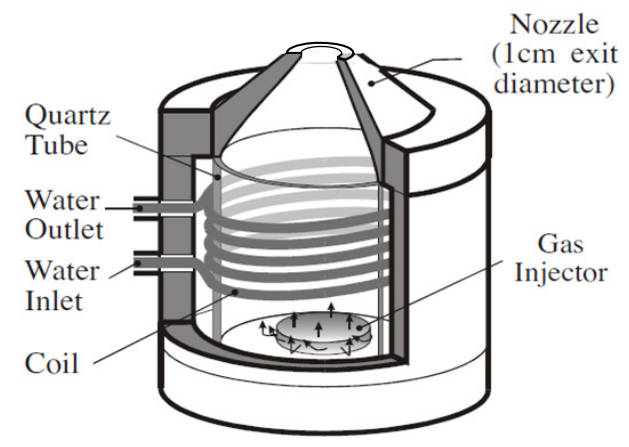

Figure 2: Plasma torch head and nozzle assembly. The gas injectors include radial, swirl and axial injectors.

\section{Experiment}

\section{A. Plasma torch facility}

The plasma torch facility used to produce the plasma at the inlet of the water-cooled test-section is a TAFA Model 66 inductively coupled plasma (ICP) torch powered by a $120 \mathrm{kVA}$ radio frequency LEPEL Model T-50-3 power supply. The power supply operates at $4 \mathrm{MHz}$ and can supply a maximum of $12 \mathrm{kV} \mathrm{DC}$ and $7.5 \mathrm{~A}$ to the oscillator plates. Details of the plasma torch facility are provided in previous publications: see Refs. [16] and [22]. The plasma at the exit of the torch is at atmospheric pressure. For the experiments presented here, a 1-cm diameter exit nozzle was used. Figure 2 shows a schematic of the facility. Calibrated flow meters are used to control the mass flow rate of each gas through the system. A $\mathrm{CO}_{2} / \mathrm{Ar}$ gas mixture was used for these measurements. The flowrates were $2.07 \pm .04 \mathrm{~g} / \mathrm{s}$ (70 slpm) for argon and $0.467 \pm .002 \mathrm{~g} / \mathrm{s}(14.2 \mathrm{slpm})$ for $\mathrm{CO}_{2}$. The $\mathrm{CO}_{2} / \mathrm{Ar}$ was premixed before being injected into the torch. $5 / 8$ of this mixture was injected via the radial injectors and $3 / 8$ via the swirl injectors. This combination was found to yield a stable plasma with a smooth temperature profile. Temperature measurements obtained by measuring the absolute emission from lines of atomic carbon $(833 \mathrm{~nm})$, oxygen $(777 \mathrm{~nm})$ and argon $(750 \mathrm{~nm})$. This is done using a visible spectrometer (Acton SpectraPro 500i) and imaging setup. A filter is installed in the optical path to suppress higher order interferences within the spectrometer. The imaging system makes use of parabolic mirrors and a periscope to image the spectrometer slit across the jet profile. Intensity measurements are Abel-inverted to provide spatially resolved intensity measurements. The procedure for these emission-based temperature measurements is documented in several references. ${ }^{16,17,23}$ The analysis relies upon the assumption of thermochemical equilibrium - an assumption that must be verified and that will be discussed further in the Results section.

\section{B. VUV spectrometer}

The spectrometer and imaging system used for VUV measurements are shown in Figure 3. The spectrometer is a McPherson spectrometer (Model 218) designed for VUV measurements. The grating used has 2400 grooves $/ \mathrm{mm}$ and is blazed at $150 \mathrm{~nm}$. The slit function has an approximate FWHM of $0.15 \mathrm{~nm}$ and is shown in Figure 4 for reference. An imaging box is attached to the spectrometer. Two spherical mirrors of $50 \mathrm{~cm}$ focal length were inserted into this box and used to image the slit onto the torch - the spectrometer slit and torch are located at the focus of each mirror and there is therefore no image magnification. These mirrors were coated with the Acton Optics \#1200 coating for 120 $\mathrm{nm}$. Through the use of a rotary vane roughing pump and a turbopump (80 liters/sec pumping speed), the system was pumped down to a pressure of approximately $6.0 \times 10^{-5}$ Torr for all tests. The rotary vane pump was complemented 


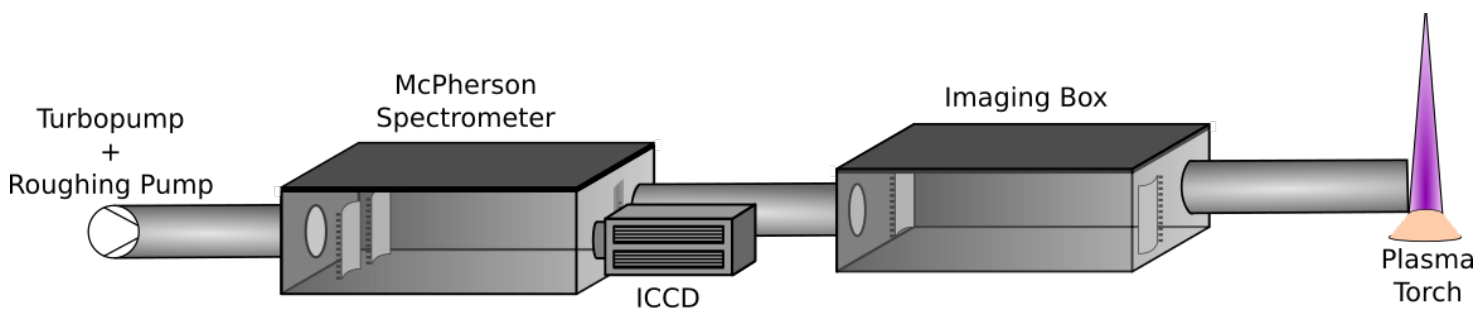

Figure 3: VUV spectrometer and imaging system used for measurements of $\mathrm{CO}(4+)$.

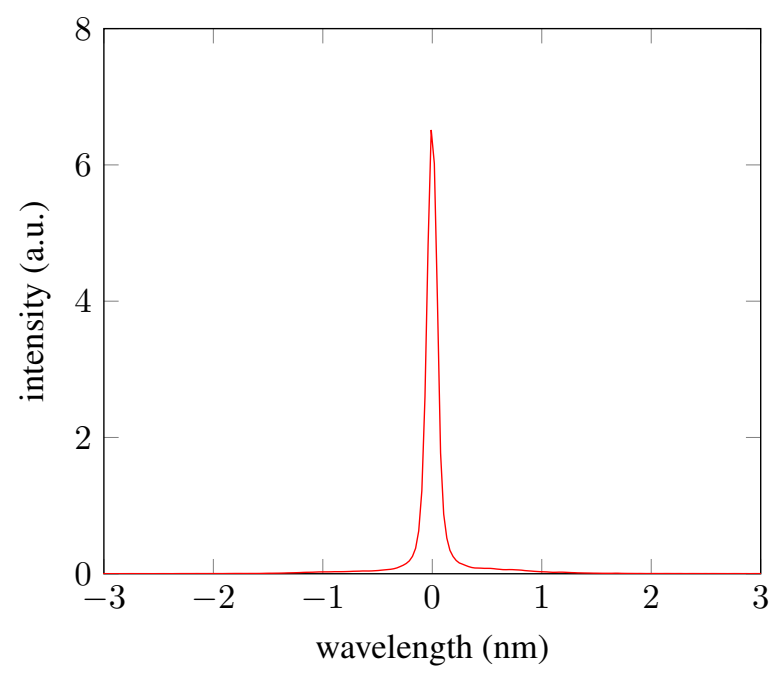

Figure 4: Measured slit function of the VUV system. The area has been normalized to one.

with a zeolite adsorbent filter to prevent oil backstreaming into the optical system. An argon purge is used to remove oxygen from the final $10 \mathrm{~cm}$ of the optical path and thus this is not under vacuum.

For calibration in absolute intensity, an argon discharge was used that is calibrated and traceable to NIST standards. ${ }^{24}$ In order to calibrate our system using this discharge, a modification of the system was required. The final $10 \mathrm{~cm}$ of the imaging line was replaced with an adaptor to mount the system directly to the discharge housing. The system aperture for the argon discharge calibration was different from the system aperture used in the plasma torch measurements. Given that all dimensions are known, this difference was calculated and taken into account in the measurements (see Appendix A). Another option would have been to install a diaphragm in the imaging box to serve as the limiting aperture of the system - thus eliminating this variable between the argon discharge calibration and plasma torch measurements. However, this would require reducing the signal-to-noise ratio and was therefore avoided.

\section{Results}

\section{A. temperature profile}

Figure 5 shows the temperature obtained by measuring the absolute atomic emission from Argon $(750 \mathrm{~nm}), \mathrm{C}(833$ $\mathrm{nm})$ and $\mathrm{O}(777 \mathrm{~nm})$. Note that the uncertainty on the atomic oxygen temperature measurement is much smaller than the $\mathrm{Ar}$ and $\mathrm{C}$ temperature measurements due to the rather high intensity of this feature under the given conditions. The uncertainty of the oxygen temperature profile is on the order of $+/-30 \mathrm{~K}$ near the jet centerline. The uncertainty bars for the Argon and Carbon measurements were about +/- $200 \mathrm{~K}$ to $+/-300 \mathrm{~K}$. Reference [16] contains a detailed discussion of the uncertainty analysis that was used for these measurements. The maximum discrepancy between the Argon and Oxygen temperature profiles, for $r<0.2 \mathrm{~cm}$ where the signal remains reasonably certain, is approximately $200 \mathrm{~K}$ and 


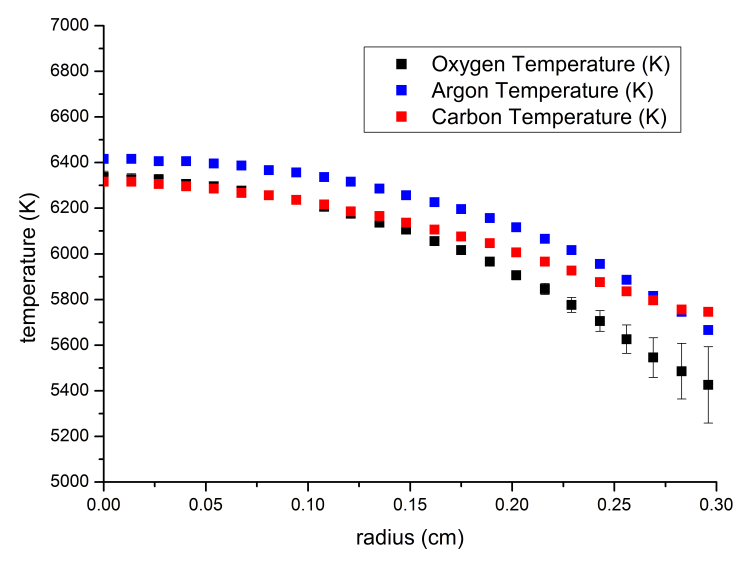

Figure 5: Temperatures obtained by measuring absolute emission from several atomic features. Only the uncertainty bars for the atomic Oxygen measurements are shown. Those of the Argon and Carbon measurements were about +/$200 \mathrm{~K}$ to $+/-300 \mathrm{~K}$.

around $100 \mathrm{~K}$ for the Carbon and Oxygen temperature profiles. The temperature measurements are therefore consistent with one another to within their respective uncertainties. Therefore, the torch profile is considered to be described by a single temperature. The high operating pressure means that thermochemical equilibrium is to be expected, and has been extensively verified in the case of air plasmas. ${ }^{16}$ Therefore, in the analysis that follows, the plasma is considered to be in thermochemical equilibrium. The temperature of the atomic oxygen line will be used for the subsequent analysis of the $\mathrm{CO}(4+)$ emission spectra because it supplies the most accurate measure of temperature.

\section{B. CO 4th positive spectra}

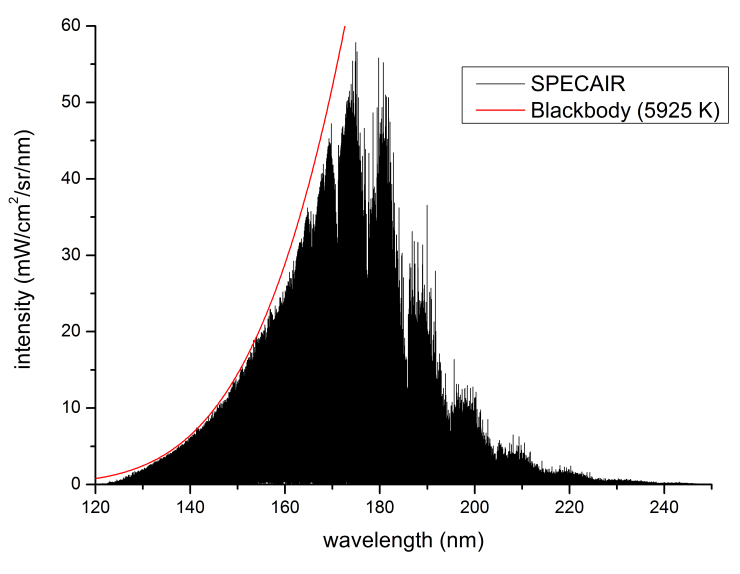

(a)

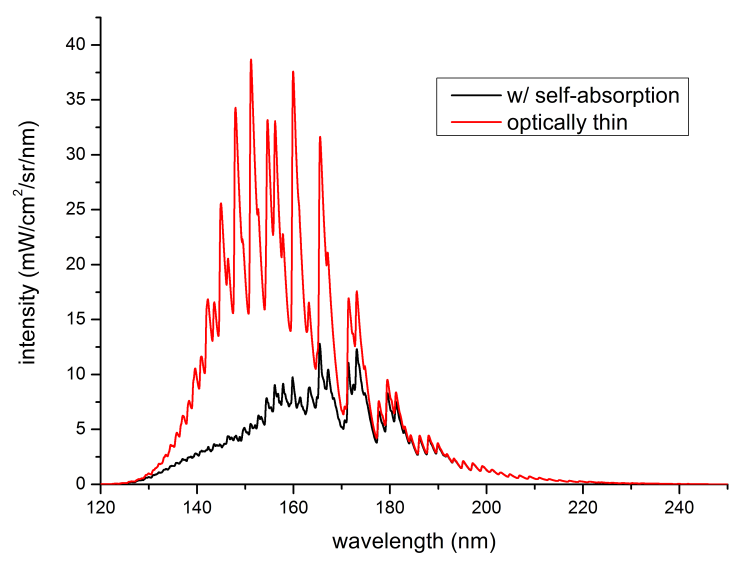

(b)

Figure 6: a) Theoretical spectra without slit function calculated for experimental conditions. The blackbody intensity curve, calculated for a temperature of $5925 \mathrm{~K}$, is shown for comparison and represents the blackbody limit below approximately $170 \mathrm{~nm}$. b) Optically thin vs optically thick spectra, calculated for experimental conditions. Below approximately $180 \mathrm{~nm}$, the CO spectrum becomes optically thick.

For visible measurements, where the plasma is optically thin, the intensity measured across the jet profile can be Abel-inverted to produce the local, spatially resolved emission. As Fig. 6 shows, experimental spectra are close to 
being blackbody limited in certain regions of the VUV spectrum. Note that the blackbody limit is not at the centerline temperature of the plasma. Though Abel-inversion of optically thick spectra is possible, the analysis becomes significantly more complex. ${ }^{25}$ Furthermore, Abel-inversion was not required for validation of radiative models. To produce a theoretical spectra for comparison with experiment, we solved the radiative transport equation across the jet profile. This was done by first subdividing the line-of-sight domain, which corresponds to the radial profile of the jet, into several sublayers. Within each sublayer $i$, a temperature $T_{i}$ and $\mathrm{CO}$ mole fraction $\chi_{i}$ were assigned based upon the measured temperature profile (Fig. 5) and assuming thermochemical equilibrium. The emission spectrum along the line-of-sight was computed using the radiation code SPECAIR ${ }^{17,26}$ with the Einstein coefficients calculated according to the aforementioned electronic transition moments taken from either DeLeon, Kirby or Spielfiedel. ${ }^{12-15}$ The final intensity $I$ was then convolved with the slit function to produce the final spectra for comparison with experimental measurements.

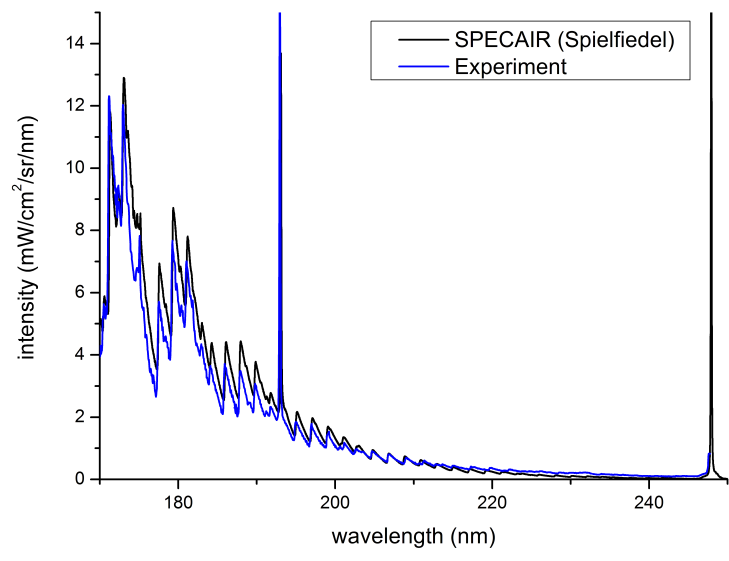

(a)

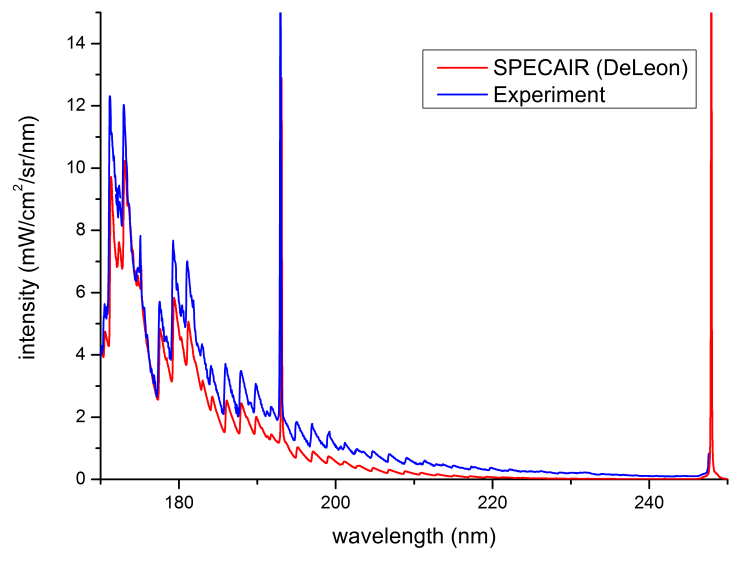

(b)

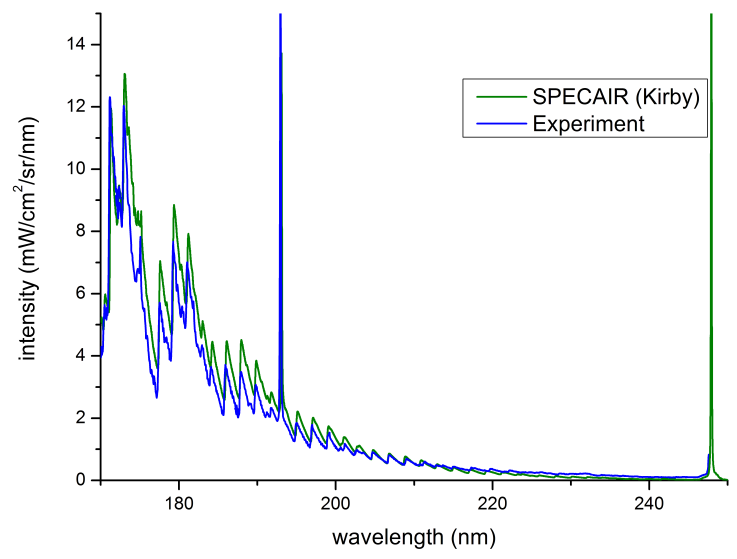

(c)

Figure 7: Comparison between experimental measurements and the spectra computed with SPECAIR using the electronic transition moments from a) Spielfiedel et al, b) DeLeon and c) Kirby and Cooper.

Figure 7 shows the VUV/UV spectrum measured in the plasma torch facility, and the comparison with the three models considered. In addition to $\mathrm{CO}(4+)$ emission, there are $\mathrm{C}$ features at $193 \mathrm{~nm}$ and $248 \mathrm{~nm}$. The agreement between the experimental measurements and the Spielfiedel and Kirby models is quite good. However, some fluctuations in our calibration measurement of approximately $30 \%$ have been noted. The source of the fluctuation is under investigation. In consideration of this fluctuation, we can currently conclude that the models of Spielfiedel and Kirby \& Cooper can plausibly reproduce the data to within this accuracy. Resolution of the fluctuations may lead to more 
definitive conclusions.

\section{Conclusion}

We have presented spectrally resolved measurements of the $\mathrm{CO}(4+)$ system down to $170 \mathrm{~nm}$ under equilibrium conditions. The preliminary results presented here indicate good agreement between the Spielfiedel and Kirby models. A discrepancy was observed relative to the computed spectrum when using the electronic transition dipole moment of DeLeon. Future work is focused on achieving fully calibrated measurements down to $150 \mathrm{~nm}$ and on resolving the aforementioned fluctuations in our calibration measurement. Absolute intensity measurements at low wavelengths are currently confounded by the presence of stray light in the spectrometer. Because the Argon mini-arc calibration source experiences a significant decrease in signal at low wavelengths, scattered light from higher wavelengths are of the same order as the calibration signal. Future work will introduce a low-pass filter to remove this effect and allow for accurate calibration. Oxygen absorption in the purged section of the imaging system at 1 atm pressure may also play a role in preventing making calibrated measurements down to $150 \mathrm{~nm}$, though it is uncertain if this is indeed a problem. Reducing sources of absorption in the purged section will be examined. The end goal should provide more conclusive data for accurate models for simulation of $\mathrm{CO} 4$ th Positive radiation.

\section{Acknowledgments}

We would like to acknowledge the technical contributions of Richard Morgan, Carolyn Jacobs and Umar A Sheikh who either participated in the development of this VUV system or provided technical advice in its design. The VUV spectrometer was obtained as part of an International NASA Space Act Agreement for Equilibrium radiation measurements. Augustin Tibère-Inglesse was supported by CIFRE grant number 42701092/20160218/JSE with Airbus Safran Launchers (technical monitor: Laurent Visconti).

\section{References}

${ }^{1}$ A.M. Brandis, C.O. Johnston, B.A. Cruden, D.K. Prabhu, A.A. Wray, Y. Liu, D.W. Schwenke, and D. Bose. Validation of CO 4th positive radiation for mars entry. Journal of Quantitative Spectroscopy and Radiative Transfer, 121:91 - 104, 2013.

${ }^{2}$ Brett A. Cruden, Dinesh Prabhu, and Ramon Martinez. Absolute radiation measurement in Venus and Mars entry conditions. Journal of Spacecraft and Rockets, 49(6):1069-1079, 2016/08/31 2012.

${ }^{3}$ W.C. Pitts and R.M. Wakefield. Performance of entry heat shields on pioneer venus probes. Journal of Geophysical Research, 85(A13):8333$8337,1980$.

${ }^{4}$ D. Bose, J.H. Grinstead, D.W. Bogdanoff, and M.J. Wright. Shock layer radiation measurements and analysis for mars entry. Technical report, NASA Ames Research Center, 2009.

${ }^{5}$ Jay H. Grinstead, Michael J. Wright, David W. Bogdanoff, and Gary A. Allen. Shock radiation measurements for mars aerocapture radiative heating analysis. Journal of Thermophysics and Heat Transfer, 23(2):249-255, 2018/06/05 2009.

${ }^{6}$ M.K. Lockwood et al. Systems analysis for a venus aerocapture mission. Technical Report TM-2006-214291, NASA, 2006.

${ }^{7}$ M.K. Lockwood et al. Aerocapture systems analysis for a titan mission. Technical Report TM-2006-214273, NASA, 2006.

${ }^{8}$ Jeffery L. Hall, Muriel A. Noca, and Robert W. Bailey. Cost-benefit analysis of the aerocapture mission set. Journal of Spacecraft and Rockets, 42(2):309-320, 2018/06/26 2005.

${ }^{9}$ Christopher O. Johnston, Brian R. Hollis, and Kenneth Sutton. Non-boltzmann modeling for air shock-layer radiation at lunar-return conditions. Journal of Spacecraft and Rockets, 45(5):879-890, 2018/06/05 2008.

${ }^{10}$ Christopher O. Johnston, Brian R. Hollis, and Kenneth Sutton. Spectrum modeling for air shock-layer radiation at lunar-return conditions. Journal of Spacecraft and Rockets, 45(5):865-878, 2018/06/05 2008.

${ }^{11}$ Arnold JO Whiting EE, Yen L and Paterson JA. Nonequilibrium and equilibrium radiative transport and spectra program: user manual. Technical Report NASA RP-1389, NASA Ames Research Center, Aerothermodynamics Division, 1996.

${ }^{12}$ R. L. DeLeon. CO (A-X) electric dipole transition moment. The Journal of Chemical Physics, 89(1):20-24, 1988.

${ }^{13}$ R. L. DeLeon. Erratum: CO (A-X) electric dipole transition moment [j. chem. phys. 89, 20 (1988)]. The Journal of Chemical Physics, 91(9):5859-5860, 1989.

${ }^{14}$ Kate Kirby and David L. Cooper. Theoretical study of low-lying ${ }^{1} \Sigma^{+}$and ${ }^{1} \Pi$ states of CO. II. Transition dipole moments, oscillator strengths, and radiative lifetimes. The Journal of Chemical Physics, 90(9):4895-4902, 1989.

${ }^{15}$ A. Spielfiedel, W. Tchang-Brillet, F. Dayou, and N. Feautrier. Ab initio calculation of the dipole transition moment and band oscillator strengths of the CO (A-X) transition. Astronomy and Astrophysics, 346:699-704, 1999.

${ }^{16}$ Christophe O. Laux. Optical Diagnostics and Radiative Emission of Air Plasmas. PhD thesis, Stanford University, Dept. of Mechanical Engineering, 1993.

${ }^{17} \mathrm{C}$ O Laux, T G Spence, C H Kruger, and R N Zare. Optical diagnostics of atmospheric pressure air plasmas. Plasma Sources Science and Technology, 12(2):125, 2003.

${ }^{18}$ Christophe O. Laux and Charles H. Kruger. Arrays of radiative transition probabilities for the $\mathrm{N}_{2}$ first and second positive, NO beta and 
gamma, $\mathrm{N}_{2}^{+}$first negative, and $\mathrm{O}_{2}$ Schumann-Runge band systems. Journal of Quantitative Spectroscopy and Radiative Transfer, 48(1):9 - 24, 1992.

${ }^{19}$ R. Farrenq, G. Guelachvili, A.J. Sauval, N. Grevesse, and C.B. Farmer. Improved dunham coefficients for co from infrared solar lines of high rotational excitation. Journal of Molecular Spectroscopy, 149(2):375 - 390, 1991.

${ }^{20}$ J.D. Simmons, A.M. Bass, and S.G. Tilford. The fourth positive system of carbon monoxide observed in absorption at high resolution in the vacuum ultraviolet region. Astrophysical Journal, 155:345-358, 1969.

${ }^{21}$ R. W. Field, O. Benoist d'Azy, M. Lavollée, R. Lopez-Delgado, and A. Tramer. Radiative decay rates from deperturbed v=0-7 vibrational levels of $\mathrm{CO} \mathrm{A}^{1} \Pi$ measured using synchrotron radiation. The Journal of Chemical Physics, 78(6):2838-2846, 1983.

${ }^{22}$ Megan E. MacDonald, Carolyn M. Jacobs, Christophe O. Laux, Fabian Zander, and Richard G. Morgan. Measurements of air plasma/ablator interactions in an inductively coupled plasma torch. Journal of Thermophysics and Heat Transfer, 29(1):12-23, 2014.

${ }^{23}$ B. J. McBride and S. Gordon. Computer program for calculating and fitting thermodynamic functions. Technical report, NASA RP-1271, 1992.

${ }^{24}$ Jules Z. Klose, J. Mervin Bridges, and William R. Ott. Radiometric calibrations of portable sources in the vacuum ultraviolet. Journal of Research of the National Bureau of Standards, 93(1):21-39, 1988.

${ }^{25}$ Stephen J. Young. Iterative abel inversion of optically thick, cylindrically symmetric radiation sources. Journal of Quantitative Spectroscopy and Radiative Transfer, 25(5):479 - 481, 1981.

${ }^{26}$ SPECAIR, Software Package, Ver. 3.0, SpectralFit S.A.S., http: //www . spectralfit.com.

\section{Appendix A : solid angle correction factor}

Figure 8 shows the difference in system configurations between the plasma torch and mini-arc calibration measurements. The purple tube adapts directly to the imaging box under vacuum. After the measurement in the plasma torch, this tube is translated in order to adapt to the calibration source. However, the actual position of the focus does not change. The green section represents the argon purge. For the case of the torch measurements, a water-cooled piece (orange/red in the figure) is used to finally adapt the system to the plasma torch. To account for the difference in solid angle, the known dimensions were used to directly calculate the system solid angle. Note that the mini-arc source emits with an equivalent F/\# which is smaller than the system F/\#. The limiting aperture is set by the tube dimensions as diagrammed in Fig. 8. Given that:

$$
\Omega=2 \pi[1-\cos \theta]
$$

where $\Omega$ is the solid angle and $\theta$ the angle defined in Fig. 8, it follows that:

$$
\frac{\Omega_{\text {calib }}}{\Omega_{\text {torch }}}=0.476
$$

The absolute intensity as reported in Fig. 7 were therefore corrected according to this difference in system aperture.

Figure 8: Top: Configuration used for VUV measurements in the torch. Bottom: Configuration used for mini-arc calibrations in the VUV. The mini-arc adapts directly to the teflon piece in green. The imaging point in both the top and bottom configurations corresponds to the intersection of the central axis (dotted line) and limiting aperture (solid line). 